\title{
Measurement of Irradiation-induced Swelling in Stainless Steels with a New Transmission Electron Microscopy Method
}

\author{
Li He ${ }^{1}$, Haixuan $\mathrm{Xu}^{2}$, Lizhen $\mathrm{Tan}^{3}$, Paul Voyles ${ }^{4}$, Kumar Sridharan ${ }^{1}$ \\ 1. Department of Engineering Physics, University of Wisconsin-Madison, Madison, Wisconsin, USA \\ 2. Department of Materials Science and Engineering, University of Tennessee, Knoxville, Tennessee, \\ USA \\ 3. Oak Ridge National Laboratory, Oak Ridge, Tennessee, USA \\ 4. Department of Materials Science and Engineering, University of Wisconsin-Madison, Madison, \\ Wisconsin, USA
}

300 series stainless steels such as $316 \mathrm{H}$ are used in nuclear reactors where they experience neutron radiation damage. An important concern here is the volume increase or swelling and associated deterioration of mechanical properties. Traditionally, transmission electron microscopy (TEM) has been used to measure void size and number density to estimate the overall volume increase. However, this technique may not yield accurate results when the voids are small or cannot be effectively resolved in TEM. Here we have developed a method to measure swelling from the valence electron density of materials using plasmon energy loss of high energy electrons in TEM. The technique is potentially applicable to any metals regardless of composition or nature of phases. Furthermore, benefiting from the well-confined TEM probe, the technique is suitable to measure nanostructure density change.

The incident electrons in TEM lose energy to specimen atoms by displacing valence electrons and creating an oscillation of electrical field (plasmon). The square of plasmon peak energy $\mathrm{E}_{\mathrm{p}}{ }^{2}$ in electron energy loss spectroscopy (EELS) is proportional to valence electron volume density [1]. A TEM sample is a thin membrane usually about $100 \mathrm{~nm}$ thick. It inevitably contains surface layer of either oxide, sample preparation damage, or other structures having different electron density from the intrinsic material. If $\rho, \mathrm{V}$, and $\mathrm{t}$ represent valence electron density, volume, and sample thickness, respectively, and $\rho_{s}, V_{s}$, and $t_{s}$ represent these quantities for the surface layer, plasmon energy is proportional to the average electron density:

$$
E_{p}^{2}=C \times \bar{\rho}=C \times \frac{\rho\left(V-V_{s}\right)+\rho_{s} V_{S}}{V}=C \times \frac{\rho\left(t-t_{g}\right)+\rho_{s} t_{s}}{t}=C\left[\rho-\left(\rho-\rho_{s}\right) \frac{t_{s}}{t}\right]
$$

By plotting and fitting $\mathrm{E}_{\mathrm{p}}{ }^{2} \sim 1 / \mathrm{t}$ linearly, the intersection to $\mathrm{y}$-axis would be proportional to $\rho$.

TEM experiments were conducted at $200 \mathrm{kV}$ using an FEI Titan S-Twin aberration corrected scanning transmission electron microscope (STEM) equipped with Gatan GIF (ER865). Figure 1(a) shows the annular dark field (ADF) image of $316 \mathrm{H}$ stainless steel irradiated with $\mathrm{Fe}^{2+}$ up to 100 dpa peak damage at $360{ }^{\circ} \mathrm{C}$. Multiple EELS line scan spectra were collected. Figure 1(b) shows an EELS spectrum fitted in $\mathrm{f}(\mathrm{x})=\mathrm{y} 0+\mathrm{A} \times \exp \left\{-\left[\left(\mathrm{x}-\mathrm{x}_{0}\right) / \text { width }\right)^{2}\right)+\mathrm{B} \times \mathrm{x}$. The fitting function is sought to match data trend near the top of plasmon peak without considering physical meaning. This yields a plasmon peak energy $E_{p}=25.95 \mathrm{eV}$.

Figure 2(a) shows a plot of $E_{p}^{2}$ of 202 EELS data points in the damaged region. From the difference of $\mathrm{E}_{\mathrm{p}}{ }^{2}$ between damaged and undamaged regions, swelling is determined and is shown in Figure 2(b), along with SRIM calculated ion concentration [2]. SRIM calculations here used $3.7 \mathrm{MeV} \mathrm{Fe}^{2+}$ into $316 \mathrm{H}$ (Fe-17Cr-10Ni-2Mo-2Mn in weight percentage, density $7.86 \mathrm{~g} / \mathrm{cm}^{3}$ ); displacement energy values $40 \mathrm{eV}$ for $\mathrm{Fe}, \mathrm{Cr}, \mathrm{Ni}, \mathrm{Mn}$, and $60 \mathrm{eV}$ for $\mathrm{Mo}$. 
Swelling in $\mathrm{Fe}^{2+}$ irradiated $316 \mathrm{H}$ up to 100 dpa at $360{ }^{\circ} \mathrm{C}$ appears to correlate inversely with ion implantation depth profile. Swelling is large at surface and gradually decreases with depth. Near the ion implantation peak the material is denser than intrinsic state, suggesting suppression of void swelling by high concentration of implanted ions. On average swelling is $0.98 \pm 0.06 \%$ at depths of $0.16-1.85 \mu \mathrm{m}$ in the damage layer.

\section{References:}

[1] Fultz B and Howe JM, Transmission electron microscopy and diffractometry of materials, (Springer) 2001 p. 177-178.

[2] Ziegler J, Ziegler MD, Biersack JP, Nuclear Instruments and Methods in Physics Research Section B 268 (2010), p. 1818.

[3] The authors acknowledge funding from the US Department of Energy Nuclear Engineering University Program (NEUP) under project Number 14-6346.

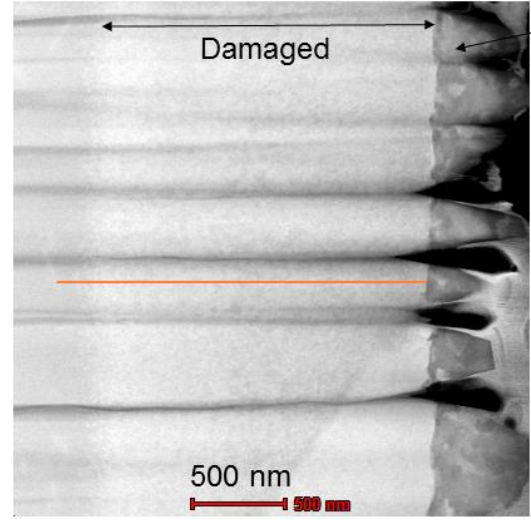

(a)

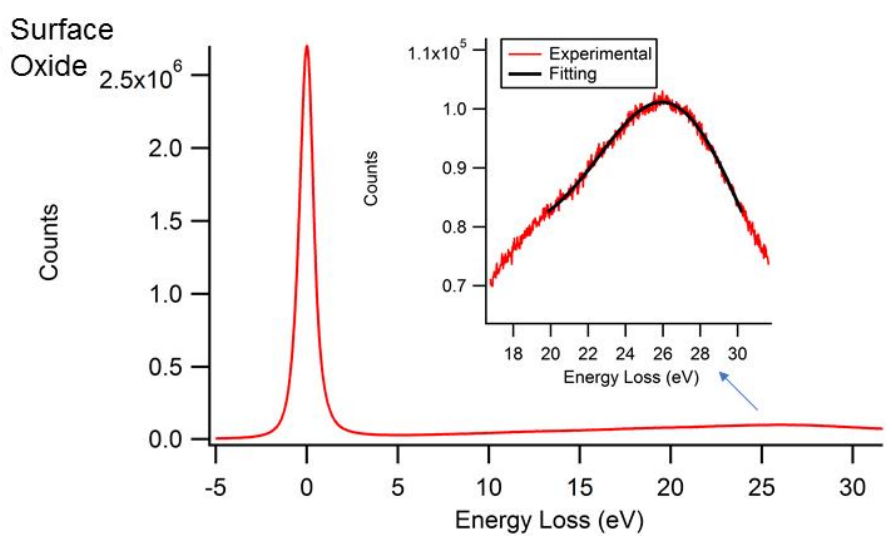

(b)

Figure 1. STEM and EELS measurement on $\mathrm{Fe}^{2+}$ irradiated $316 \mathrm{H}$ : (a) ADF image with beam semiconvergence angle $24.5 \mathrm{mrad}$ and collection angle 25-55 mrad. (b) EELS spectrum acquired on the red line at $1.84 \mu \mathrm{m}$ depth from oxide-metal interface in (a).

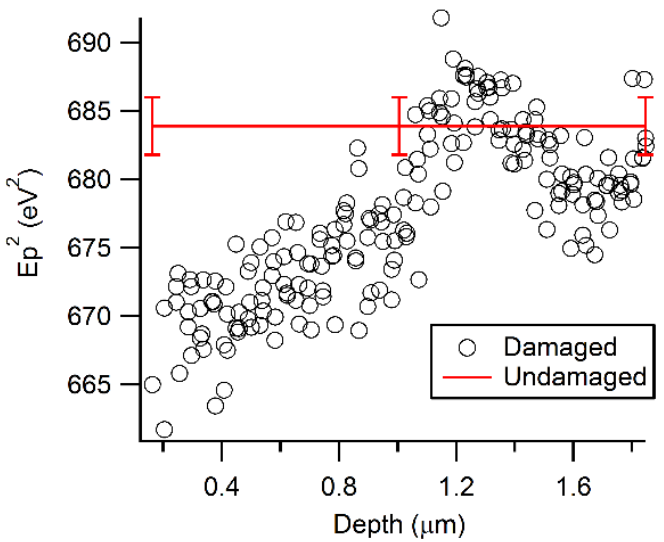

(a)

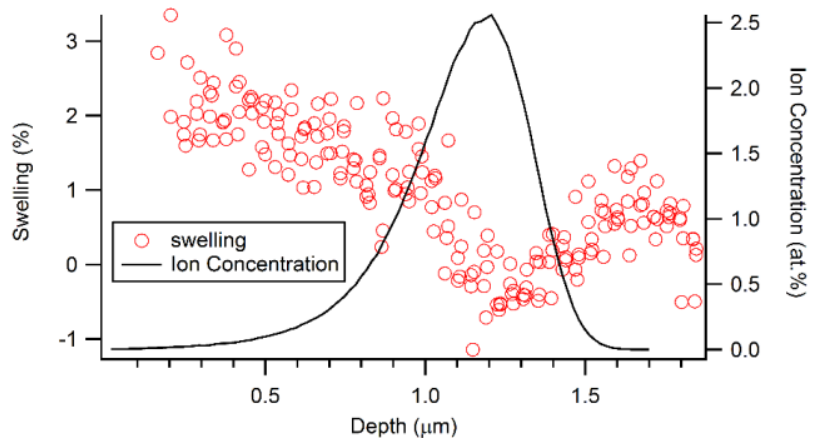

(b)

Figure 2. Swelling measurement of $\mathrm{Fe}^{2+}$ irradiated $316 \mathrm{H}$ up to $100 \mathrm{dpa}$ at $360{ }^{\circ} \mathrm{C}$ : (a) plasmon peak energy square vs. depth in the damaged region after sample thickness correction, (b) swelling depth profile. Ion concentration was obtained from SRIM calculations [2]. 